\title{
ON THE REPRESENTATION OF INTEGERS BY SUMS OF POLYNOMIALS
}

\author{
L. KUIPERS
}

All letters in this paper stand for integers.

L. K. Hua $[1]^{1}$ proved the following theorem.

TheOREM. For any positive integer a, every integer can be expressed in infinitely many ways as a sum of seven values of the cubic function

$$
f(x)=\frac{a}{6}\left(x^{8}-x\right)+x
$$

for integral values of $x$.

A. Sugar [2] improved this result. He deduced the following theorem.

TheOREM. For any $a, c$, and any $g$ prime to $a$, every integer can be expressed in infinitely many ways as a sum of five values of the function

$$
p(x)=\frac{a}{6}\left(x^{3}-x\right)+g x+c
$$

for integral values of $x$.

The last theorem now is a special case $(k=1)$ of the following theorem.

Theorem I. For any $a, c$, and $g$ prime to $a$, and any $k \geqq 1$, every integer can be expressed in infinitely many ways as a sum of $\left(4^{k}+1\right)$ values of the $\left(2^{k}+1\right)$ th degree polynomial in $x$

$$
\phi(x)=\frac{a(x+k)(x+k-1) \cdots(x-k)}{(2 k+1) !}+g x+c
$$

for integral values of $x$.

Furthermore we deduce:

TheOREM II. For any $g$ and $c$, and any $k \geqq 1$, every integer can be expressed as a sum of $4^{k}$ values of the $\left(2^{k}+1\right)$ th degree polynomial in $x$

$$
\phi(x)=\frac{(x+k)(x+k-1) \cdots(x-k)}{(2 k+1) !}+g x+c
$$

for integral values of $x$.

Received by the editors October 7, 1950.

1 Numbers in brackets refer to the literature cited at the end of the paper. 
Proof. Let $\psi(x, k)$ be the $(2 k+1)$ th degree polynomial in $x$

$$
\frac{a(x+k)(x+k-1) \cdots(x-k)}{(2 k+1) !} \text { or } a C_{x+k, 2 k+1} \text {, }
$$

then $\phi(x)$ is the polynomial $\psi(x, k)+g x+c$. If $\Delta_{x} f(x)$ denotes the difference $f(x+1)-f(x)$, then from the well known identity $C_{n, k}-C_{n-1, k}$ $=C_{n-1, k-1}$ we have:

$$
\begin{aligned}
& \Delta_{x} \phi(x-1)=\Delta_{x} \psi(x-1, k)+g, \\
& \Delta_{x}^{2} \phi(x-1)=\Delta_{x}^{2} \psi(x-1, k)=\psi(x, k-1) \text {, } \\
& \Delta_{x}^{4} \phi(x-2)=\Delta_{x}^{4} \psi(x-2, k)=\psi(x, k-2) \text {, } \\
& \Delta_{x}^{2 k-2} \phi(x-k+1)=\psi(x, 1) \text {, } \\
& \Delta_{x}^{2 k} \phi(x-k)=\psi(x, 0)=x a .
\end{aligned}
$$

On the other hand, $\Delta_{x}^{2 k} \phi(x-k)$ can be expressed in the form

$$
\sum_{l=0}^{2 k}(-1)^{l} C_{2 k, l} \phi(x+k-l),
$$

and this expression can be written as

$$
\begin{aligned}
& \sum_{l=0}^{2 k} C_{2 k, 2 l} \phi(x+k-2 l)-\sum_{l=1}^{k} C_{2 k, 2 l-1} \phi(x+k-2 l+1) \\
& =\sum_{l=0}^{2 k} C_{2 k, 2 l} \phi(x+k-2 l)+\sum_{l=1}^{k} C_{2 k, 2 l-1} \phi(-x-k+2 l-1) \\
& \left.\quad-2 c \sum_{l=1}^{k} C_{2 k, 2 l-1} \quad \text { (as follows from } \phi(-x)=-\phi(x)+2 c\right) \\
& =\sum_{l=0}^{2 k} C_{2 k, 2 l} \phi(x+k-2 l)+\sum_{l=1}^{k} C_{2 k, 2 l-1} \phi(-x-k+2 l-1)-2^{2 k} c \\
& =S(x)-2^{2 k} c \text {, say. }
\end{aligned}
$$

It is obvious that $S(x)$ is the sum of $C_{2 k, 0}+C_{2 k, 1}+\cdots+C_{2 k, 2 k}=4^{k}$ values of the polynomial $\phi(x)$. Furthermore, for any integer $x$ we have

$$
\phi(x)=\psi(x, k)+g x+c \equiv g x+c(\bmod |a|) .
$$

If now $n$ is an arbitrary integer, then, as $g t+c$ runs through a complete set of residues mod $|a|$ when $t$ does, there exist infinitely many integers $t$, such that 


$$
n-2^{2 k} c \equiv g t+c(\bmod |a|),
$$

so that from (1)

$$
n-2^{2 k} c \equiv \psi(t, k)+g t+c(\bmod |a|),
$$

or

$$
n=\phi(t)+m a+2^{2 k} c=\phi(t)+S(m) .
$$

This completes the proof of Theorem I.

For $a=1$ the identity $\Delta_{x}^{2 k} \phi(x-k)=x a$ becomes

$$
x+2{ }^{2 k} c=S(x)
$$

and $S$ runs through all integers when $x$ does. This proves Theorem II.

\section{LITERATURE}

1. L. K. Hua, On the representation of integers by sums of seven cubic functions, Tohoku Math. J. vol. 41 (1935-1936) pp. 361-366.

2. Alvin Sugar, On a result of Hua for cubic polynomials, Bull. Amer. Math. Soc. vol. 47 (1941) pp. 164-165.

UNIVERSITY OF INDONESIA 